\title{
Control de posición de un sistema hidráulico mediante un controlador por realimentación de estados
}

Fernando Martínez*

Edwin Cortes**

Edgar Moreno ${ }^{* * *}$

\section{Resumen}

En este artículo se presenta el diseño de un controlador para un sistema hidráulico compuesto por una servoválvula, un sensor de posición y unidad lineal de $200 \mathrm{~mm}$ de carrera, que usa realimentación de estados. Para esto, se obtuvieron muestras de los valores de la salida y entrada real del sistema, mediante una tarjeta de adquisición de datos de National Instruments (USB-6211) y el Data Acquisition Toolbox de Matlab. Con los datos adquiridos y el uso del System Identification Toolbox de Matlab, se estableció un modelo adecuado que describe un comportamiento similar al del sistema hidráulico real, para el cual es diseñado un controlador por realimentación de estados y ubicación de polos, cuya función es controlar el sistema hidráulico a través de la tarjeta de adquisición de datos y el PC en tiempo real.

Palabras clave: adquisición de datos, identificación de sistemas, retroalimentación de estados, ubicación de polos

\section{Abstract}

This article shows the controller design for a hydraulic system, which consists of a servo-valve, a position sensor, a linear cylinder, a filter, and a hydraulic unit. Data samples are obtained from the real system input and output using a data acquisition board from National Instruments (USB-6211) and Matlab's Data Acquisition Toolbox. These data are processed with the System Identification Toolbox of Matlab, to provide a suitable model that describes a similar behavior to the real hydraulic system, for which is designed a state feedback controller and root locus, that works to control the hydraulic system through the data acquisition board and a PC on real time.

Keywords: Data Acquisition, Root Locus, State Feedback, System Identification

* Docente en la Universidad Distrital Francisco José de Caldas. Contacto: fmartinezs@udistrital.edu.co

** Tecnólogo en Electricidad de la Universidad Distrital Francisco José de Caldas. Contacto: edwincortes_07@hotmail.com, edfemoro@gmail.com

*** Tecnólogo en Electricidad de la Universidad Distrital Francisco José de Caldas. 




\section{Introducción}

El presente documento está divido en seis secciones. La segunda y tercera tratan de la adquisición de datos e identificación de un modelo del sistema hidráulico respectivamente; la cuarta, sobre el diseño del controlador por realimentación de estados y los resultados del controlador en el modelo. En la quinta sección se presentan los resultados de la utilización del controlador en el sistema real. Finalmente, se presentan resultados y se dan conclusiones del proyecto.
En este artículo se muestra el diseño de un controlador por realimentación de estados para un sistema hidráulico, como se muestra en la figura 1. Se trata de controlar una unidad lineal de 0,2 metros de carrera, cuyo elemento final de control es una servoválvula distribuidora $4 / 3$ vías, la cual se controla mediante el voltaje de la tarjeta de adquisición de datos. Para la realimentación se tiene un sensor de desplazamiento que entrega una señal de voltaje de 0 a $10 \mathrm{~V}$. También se tiene una unidad abastecedora de energía, un filtro de presión y manómetros (Martínez y Rairán, 2001).

Figura 1. Sistema hidráulico

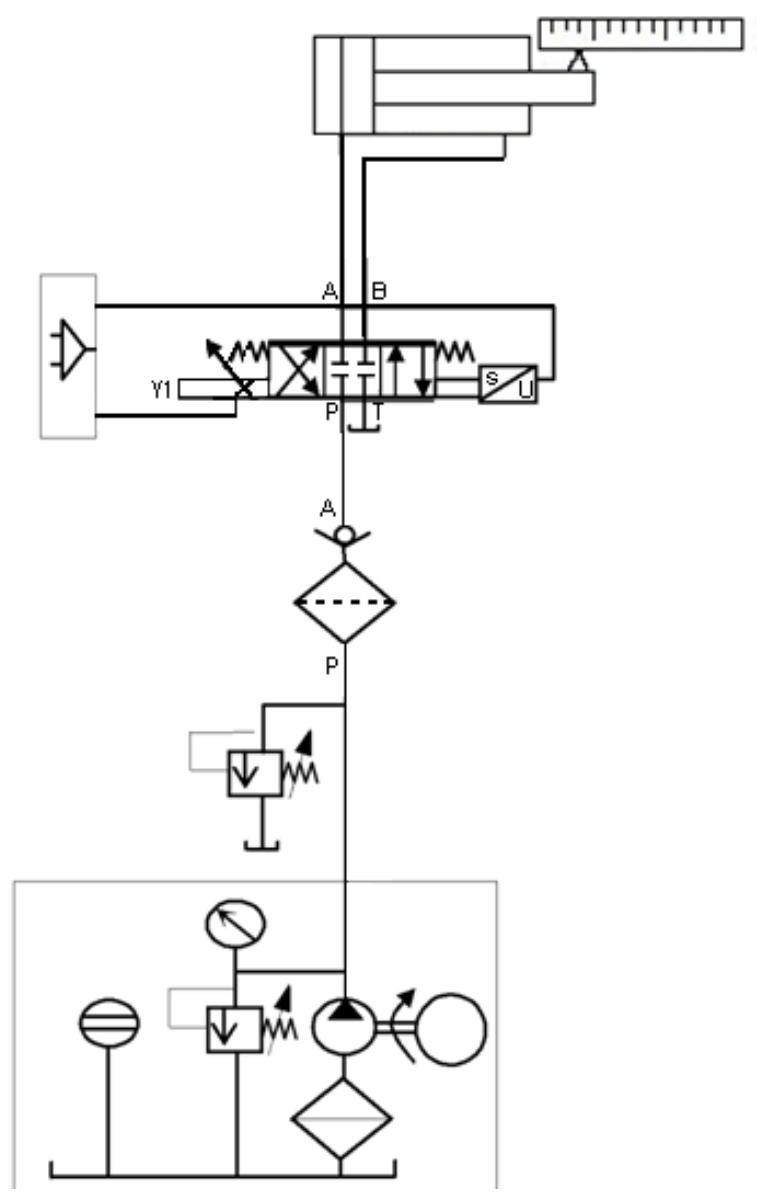

\author{
Unidad linealL, 200 \\ $\mathrm{mm}$ de carrera \\ Válvula proporcional \\ de $4 / 3$ vias.
}

Filtro de presión. Válvula reguladora
de presión.

Grupo Hidraulico 


\section{System Identification Toolbox}

La herramienta de Matlab que realiza la identificación se denomina System Identification Toolbox (SIT). Esta caja de herramientas de identificación de sistemas permite construir modelos matemáticos de sistemas dinámicos lineales, que parten de la medición de los datos de entrada-salida del sistema.

Este enfoque basado en los datos ayuda a describir los sistemas que no son fácilmente modelados desde leyes físicas (caudal-presión, presión-fuerza, velocidad-fuerza) o de las especificaciones, tales como los procesos químicos y la dinámica del motor. También ayuda a simplificar los modelos detallados por principios físicos, tales como modelos de elementos finitos de las estructuras y los modelos de dinámica de

\section{Espacio-estados}

El estado en las ecuaciones de variable-estado forman un espacio lineal, llamado espacio. Por lo tanto, el diseño usando ecuaciones de variables-estado es también denominado llamada diseño por espacio-estado.

$$
\begin{aligned}
& \dot{x}=A x+B u \\
& y=C x+D u \\
& \text { (Ecuación 1) }
\end{aligned}
$$

\section{Ubicación de polos}

Al considerar la ecuación 1 , la técnica conocida de ubicación de polos asume que todas las variables de estado son accesibles y medibles mediante la realimentación. Si el sistema en cuestión es de estado completamente controlable, los polos del sistema en lazo cerrado se pueden ubicar en cualquier posición deseada haciendo uso de vuelo, mediante el ajuste de modelos más sencillos a sus respuestas simuladas (Ljung, 2008). Los modelos obtenidos con el siT son muy adecuados para la simulación, la predicción y diseño de sistemas de control (Ljung, 2008).

Estructuras de modelos disponibles se incluyen en los modelos de procesos de orden inferior, las funciones de transferencia, el modelo de espacio-estados, los modelos lineales con no linealidades estáticas en las entradas o salidas, y los modelos no lineales autorregresivos. Si se cuenta con un modelo matemático de la dinámica del sistema, se pueden ajustar estos parámetros para adaptarse mejor a los datos experimentales (Ljung, 2008).

En la ecuación 1, A, B, y C son respectivamente las matrices $\mathrm{n} \times \mathrm{n}, \mathrm{n} \times 1$ y $1 \times \mathrm{n}$. La constante $\mathrm{D}$ es un escalar. La ecuación 1 se dice es controlable si se puede transferir cualquier estado a cualquier otro estado en un tiempo finito, aplicando una entrada. La ecuación dice ser observable si se determina el estado inicial desde el conocimiento de la entrada y la salida sobre un intervalo finito de tiempo.

una matriz de ganancias de la realimentación del estado (Katsuhiko, 1998).

Si $(\mathrm{A}, \mathrm{b})$ es no controlable, entonces la matriz característica es no singular y la ecuación correspondiente no puede ser transformada de forma controlable (Rairán, Guerrero y Mateus, 2010). 


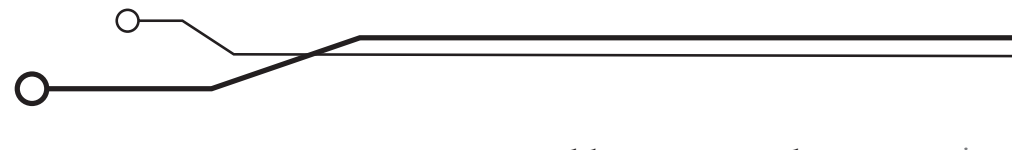

En este caso, no es posible asignar arbitrariamente cualquier valor de polos (A-bK). Sin embargo, es posible asignar algunos de ellos.

Ahora, suponiendo que los polos deseados en lazo cerrado estén en $s=\mu 1, s=\mu 2, \ldots, s=\mu 3$, y una vez seleccionada una matriz de ganancias apropiada para una realimentación del estado, es posible obligar al sistema a que tenga los polos en lazo cerrado en las posiciones deseadas, bajo la condición de que el sistema original sea de estado completamente controlable (Katsuhiko, 1998).

\section{Adquisición de datos del sistema hidráulico}

Figura 2. Componentes de un típico sistema de adquisición de datos

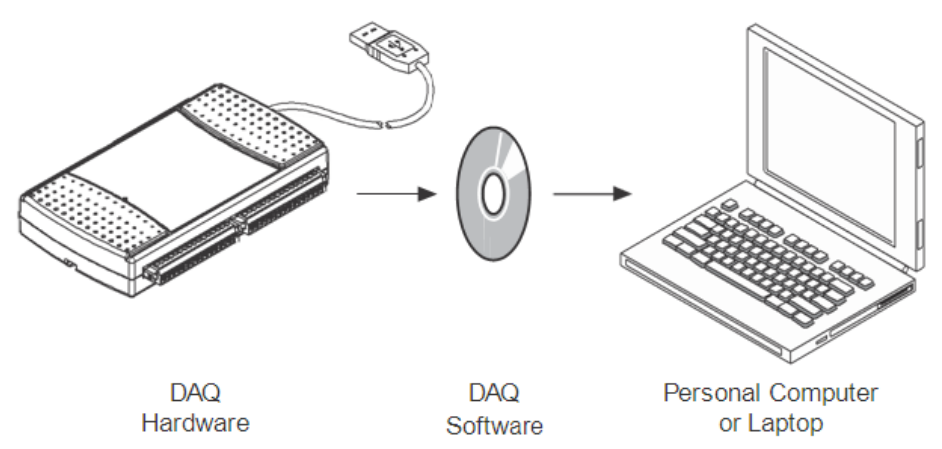

Fuente: National Instruments (2009, p. 21)

Para realizar la adquisición de datos, mostrada en la figura 2, se utiliza el hardware NI-USB 6211 de National Instruments. El software para la tarjeta es NI-DAQmx 8.9.5 DVD; y el software empleado en el PC es Matlab R2008a, que contiene Simulink, y el Data Acquisition Toolbox, esenciales para llevar a cabo la adquisición de datos reales del sistema hidráulico. La adquisición se realiza desde la tarjeta de adquisición de datos NI USB-6211(National Instruments, 2009), con la cual se obtienen, desde Simulink, tanto la señal de control a la servoválvula (salida) como la señal del sensor de desplazamiento (entrada).

Se genera desde Simulink una señal de tensión entre: $[0 ; 10] \mathrm{V},[0 ;-10] \mathrm{V}$ o $[-10 ; 10] \mathrm{V}$, dependiendo del modelo a establecer: de avance, de retroceso o con cambios de dirección, respectivamente. La señal de control es consignada en una salida análoga referenciada de la tarjeta de adquisición, conectada a la servoválvula (salida). La señal del sensor de desplazamiento está en una entrada análoga en modo diferencial, porque esta mide la diferencia entre dos entradas análogas y permite una correcta visualización de la señal. Las entradas y las salidas análogas convierten las señales a formato digital de 16-bit, con un muestreo de 25000 muestras por tiempo de simulación.

$$
\begin{gathered}
\text { Tiempo Muestreo }=\frac{\text { Tiempo Simulación }}{\text { Número Muestras }} \\
\text { (Ecuación 2) }
\end{gathered}
$$

El tiempo de muestreo se configura en Zero-Order Hold y en Analog Input. En la figura 3 se muestra el diagrama de adquisición en el que se genera la señal de control de la servoválvula, y se adquieren los datos del sensor de posición y de control. 
Figura 3. Montaje del sistema de adquisición de datos en Simulink

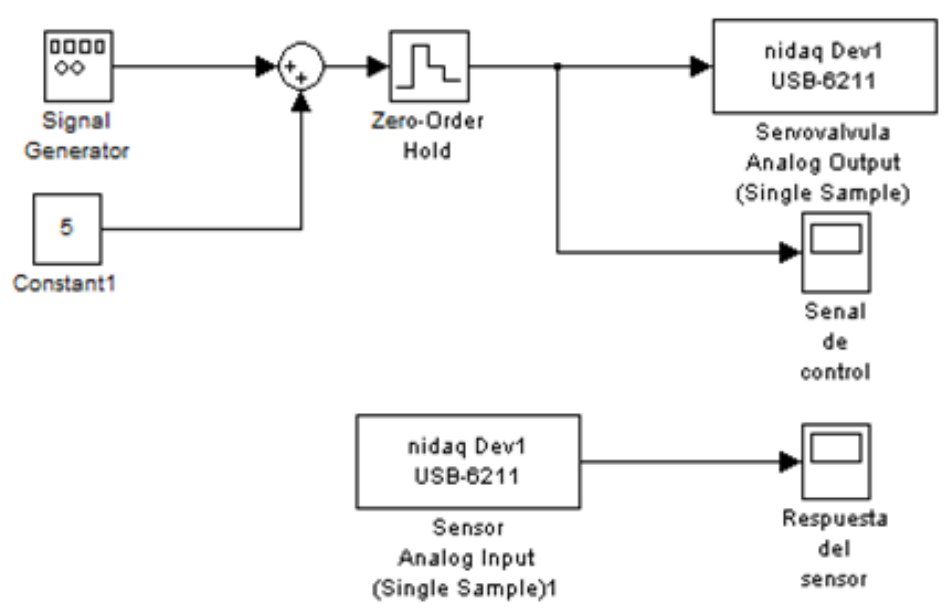

Fuente: elaboración propia

Se alimenta la servoválvula con diferentes señales (diente de sierra, seno, cuadrada y random), para observar cada uno de los comportamientos e iniciar la caracterización más apropiada del sistema.

\section{Identificación de un modelo del sistema hidráulico}

Para la identificación se necesitan los datos adquiridos anteriormente. Al estimar el modelo, el Toolbox habilita la opción model output, que permite observar la respuesta adquirida, la respuesta del modelo y el porcentaje de coincidencia o best fit. El porcentaje de coincidencia está relacionado con el criterio de información de Akaike (AIC) y el error final de la predicción de Akaike (FPE, por su sigla en inglés). Este criterio proporciona una medida de la calidad del modelo mediante la simulación de la situación en que se prueba el modelo en un conjunto de datos. Después de calcular varios modelos diferentes, se comparan con este criterio y -según la teoría de Akaike- el modelo más preciso tendría la menor FPE (MathWorks).

$$
F P E=V\left(\frac{1+d / N}{1-d / N}\right)
$$

(Ecuación 3)
En la ecuación 3, V es la función perdida, d es el número de parámetros estimados $\mathrm{y} \mathrm{N}$ es la cantidad de datos utilizados en la estimación (MathWorks). Es necesario seleccionar un modelo del sistema hidráulico, tanto para cuando la unidad avanza como para cuando retrocede, dadas las siguientes razones:

- Existe una diferencia marcada entre el tiempo de avance (ta) y el de retroceso (tr) de la unidad lineal del sistema hidráulico, la cual no debería presentarse en un sistema lineal, pues en los dos casos la electroválvula se alimenta con la misma amplitud de voltaje. Dicha diferencia es entonces causada por la diferencia en las ir áreas interiores del cilindro, y al definir el caudal definen también la velocidad (Rairán y Urrego, 2006). 


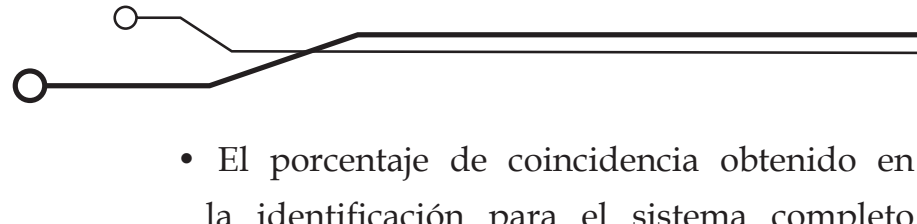
la identificación para el sistema completo los porcentajes obtenidos para los modelos de (avance y retroceso) es menor comparado con avance y de retroceso por separado.

Tabla 1. Porcentajes de coincidencia de los modelos identificados.

\begin{tabular}{|l|l|l|l|l|l|}
\hline Señal & $\mathbf{A}(\mathbf{v})$ & $\mathbf{F}(\mathbf{H z})$ & Tm (s) & Fit (\%) & Modelo \\
\hline R & 5 & 5 & $1 \mathrm{E}-4$ & 60.89 & P2Z \\
\hline R+ & 10 & 60 & $1 \mathrm{E}-4$ & 93.0 & P2Z \\
\hline R+ & 10 & 60 & $1 \mathrm{E}-4$ & 92.42 & P1Z \\
\hline R- & -10 & 60 & $1 \mathrm{E}-4$ & 89.21 & P1Z \\
\hline \multicolumn{5}{|l|}{} \\
\hline S & \multicolumn{7}{|l|}{} & & 87.49 & P2Z \\
\hline S+ & 1 & $1 E 5$ & $1 \mathrm{E}-7$ & 97.9 & P2Z \\
\hline S- & -1 & $1 \mathrm{E} 5$ & $1 \mathrm{E}-7$ & 97.87 & P2Z \\
\hline \multicolumn{5}{|l|}{} \\
\hline C & 1 & 500 & $1 \mathrm{E}-5$ & 90.36 & P2Z \\
\hline C+ & 5 & 5000 & $1 \mathrm{E}-4$ & 92.95 & P2I \\
\hline C- & -5 & 5000 & $1 \mathrm{E}-4$ & 93.13 & P2I \\
\hline \multicolumn{5}{|l}{} \\
\hline D & 5 & 100 & $1 \mathrm{E}-4$ & 86.1 & P2Z \\
\hline D+ & 1 & 9000 & $1 \mathrm{E}-5$ & 94.33 & P2I \\
\hline D- & 1 & 9000 & $1 \mathrm{E}-5$ & 95.36 & P3IZ \\
\hline
\end{tabular}

*Acá, R: random, S: seno, C: cuadrada, D: diente, +: avance, -: retroceso, P: polos, Z: ceros, I: integradores Fuente: elaboración propia

Para seleccionar un modelo adecuado se debe tener en cuenta lo siguiente:

- El porcentaje de coincidencia (Fit) debe superar el $88 \%$, según la tabla 1 .

- La respuesta de cada modelo deber ser similar a la del sistema real, al aplicar una señal de $1 \mathrm{~V}$. a lazo abierto, como se muestra en figura 4. En ocasiones, las identificaciones con mayor porcentaje no son las más apropiadas para un modelamiento del sistema ya que no tienen un comportamiento afín con el real.

Se determina que la señal seno $(97,95 \%$ y $97,87 \%$, avance y retroceso $\mathrm{r}$ /mente) es la de mayor porcentaje de coincidencia, pero al realizar el modelo en Simulink su respuesta no es la apropiada para caracterizar el sistema. Como se ve en las figuras 4 y 5 . 
Figura 4. Respuesta a lazo abierto del modelo seno de avance

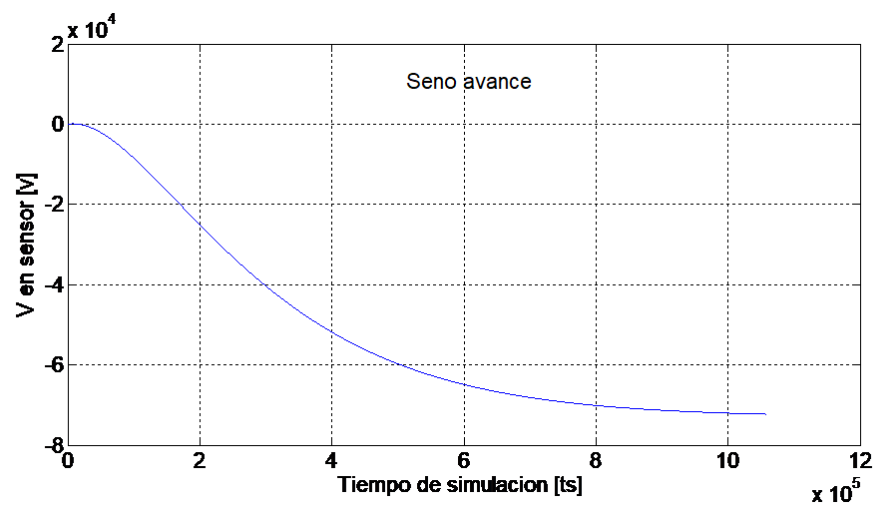

Fuente: elaboración propia

Figura 5. Respuesta a lazo abierto del modelo seno de retroceso



Fuente: elaboración propia

Figura 6. Respuesta del modelo de avance en lazo abierto

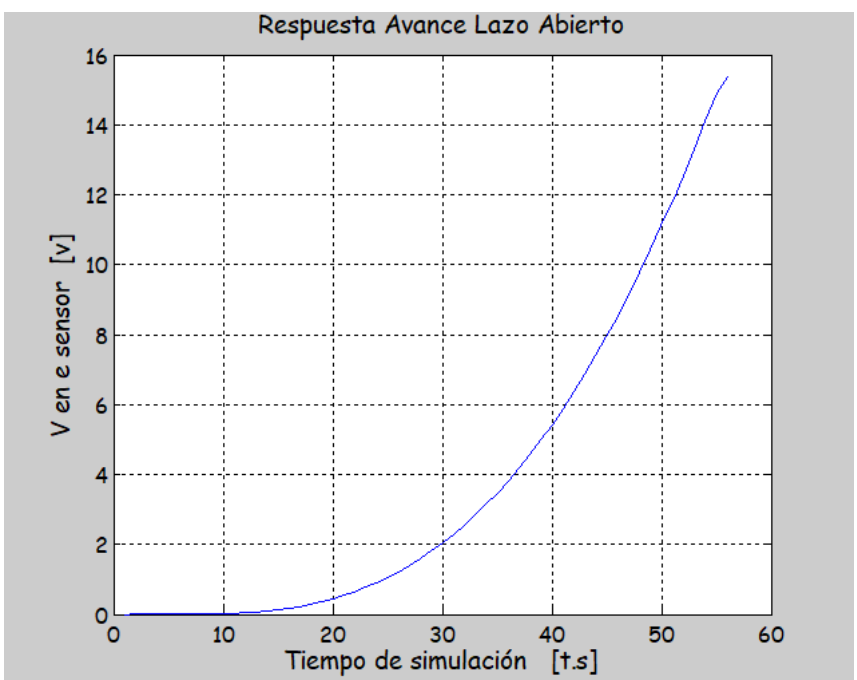

Fuente: elaboración propia 


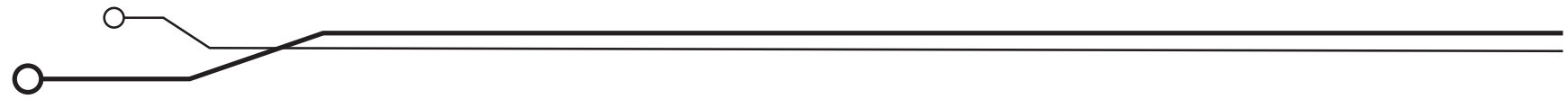

Al caracterizar los sistemas tanto de avance como de retroceso con la señal cuadrada, se conoce que el modelo está caracterizado por 2 polos y un integrador (P2I). De avance se tiene un porcentaje de coincidencia con respecto a la señal el sensor de avance $92,95 \%$ y de retroceso $93,13 \%$. En la figura 6 se muestra la respuesta del modelo escogido ante una señal de $1 \mathrm{~V}$, la cual concuerda con la señal del sistema real.

En la figura 7 se muestra la respuesta del modelo de retroceso ante una señal de $-1 \mathrm{~V}$., la cual concuerda con la respuesta del sistema real.

Figura 7. Respuesta del modelo de retroceso en lazo abierto

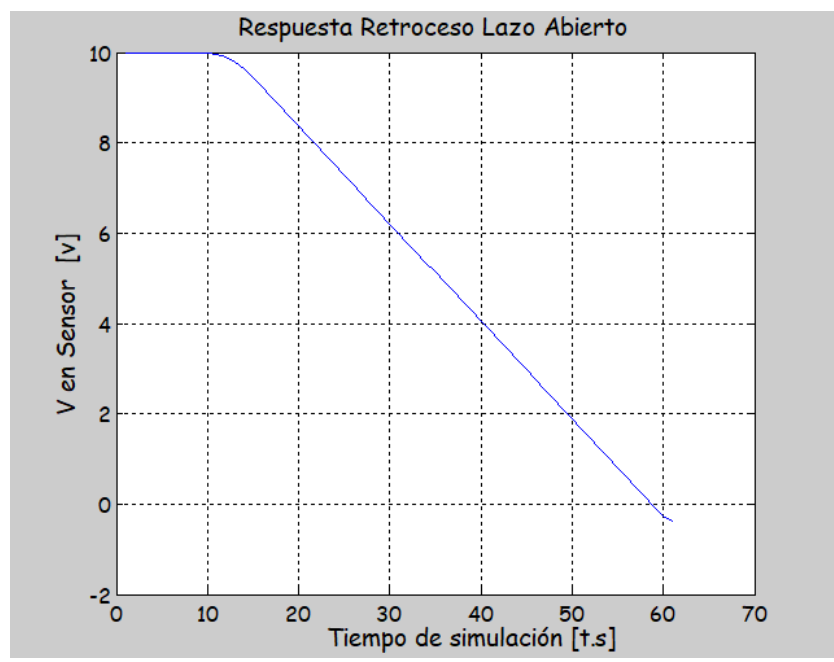

Fuente: elaboración propia

En la figura 8 se muestra la señal aplicada al sistema de avance con su respectiva respuesta.

Figura 8. Voltaje aplicado a la servoválvula y respuesta del sensor

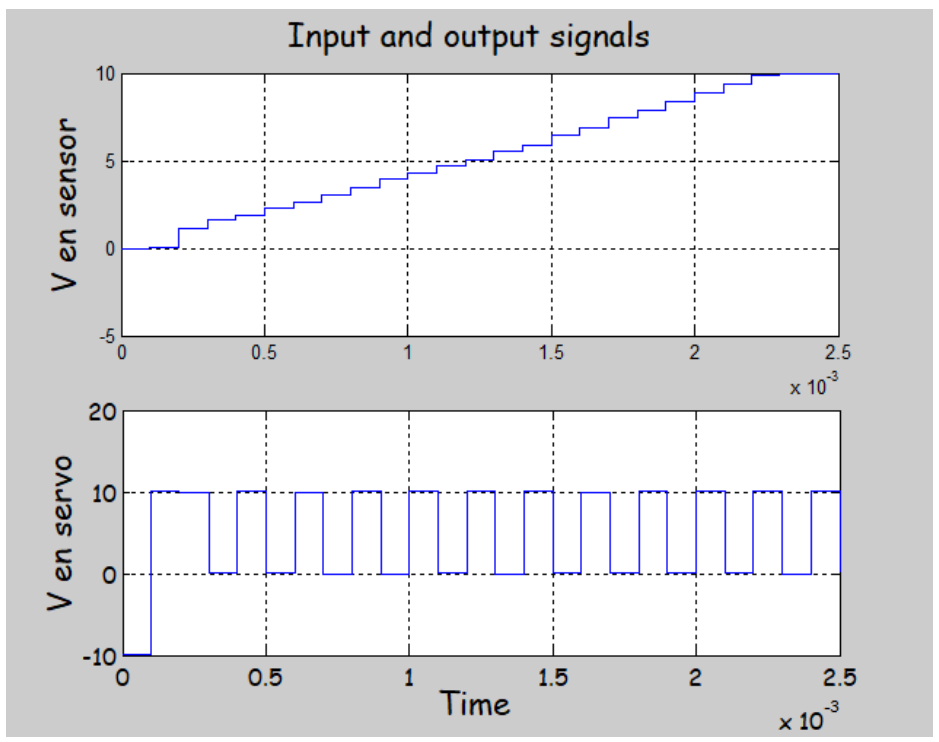


La figura 9 muestra la respuesta del sistema y del modelo con 2 polos y un integrador. Al elegir este modelo se busca que la respuesta en lazo abierto del modelo, al aplicarle una señal de $1 \mathrm{~V}$, sea parecida a la del sistema. Para esto se exporta el modelo al workspace y se halla el espacio-estados del sistema, mediante matrices con las que se puede establecer el modelo en Simulink, y así observar su respuesta ante la señal de $1 \mathrm{~V}$.

Figura 9. Respuesta del sistema y del modelo encontrado

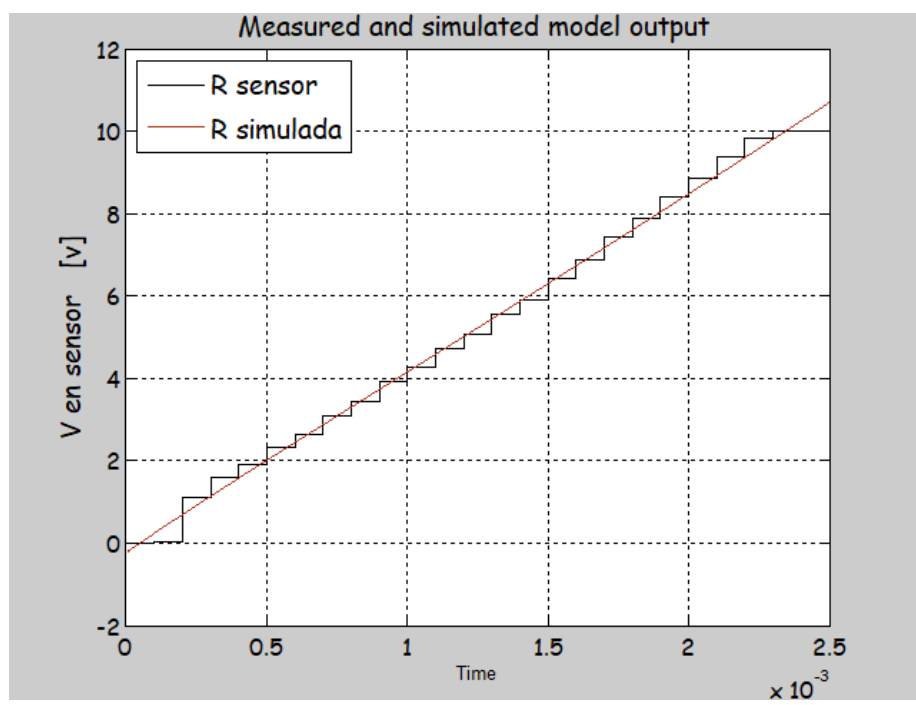

Fuente: elaboración propia

Ahora se realiza el mismo procedimiento para el modelo de retroceso. En la figura 10 se muestra la señal aplicada al sistema de retroceso con su respectiva respuesta.

Figura 10. Voltaje aplicado a la servoválvula y respuesta del sensor en retroceso






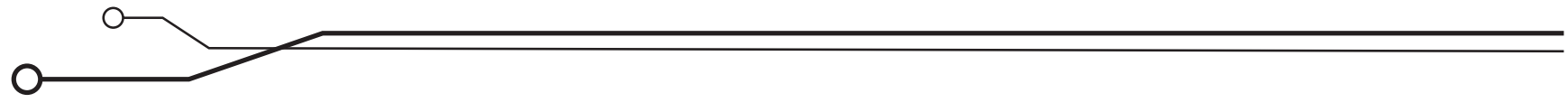

La figura 11 muestra la respuesta del sistema en retroceso y del modelo con 2 polos y un integrador.

$$
\frac{940633780.256}{s^{*}(s+1000)^{2}}
$$

(Ecuación 4)

Las funciones de transferencia de los modelos elegidos se detallan en las ecuaciones 4 y 5 .

$$
H_{s}=\frac{k}{s(s+p 1)(s+p 2)}=\frac{212827988.9475}{s(s+985.1)(s+999.9)}
$$

(Ecuación 5)

Figura 11. Respuesta del sistema y del modelo encontrado de retroceso

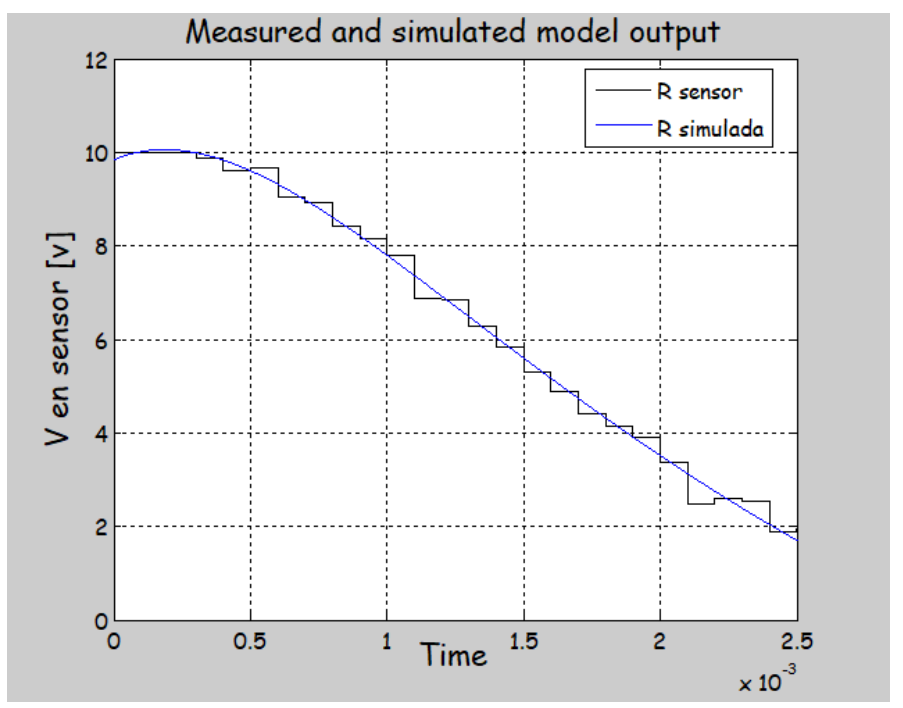

Fuente: elaboración propia

Una vez obtenidas las funciones de transferencia de los modelos, se procede a diseñar el controlador por realimentación de estados.

\section{Controlador por realimentación de estados y ubicación de polos}

Las diferentes velocidades de avance y retroceso del sistema a un mismo voltaje de alimentación hacen al sistema no lineal. Entonces, se diseña un controlador para avance y otro para retroceso, lo que permite trabajar con dos sistemas lineales. En esta sección se presenta el diseño del controlador por ubicación de polos, el cual parte de conocer el espacio-estados del modelo identificado anteriormente, en los términos de la ecuación 1. 


\section{Modelo de avance}

Para determinar las matrices del espacio-estado con Matlab se usa la función de la ecuación 4, pues ese es el modelo identificado para el avance.

$$
\begin{gathered}
A=\left[\begin{array}{ccc}
0 & 1 & 0 \\
0 & -1000 & 1 \\
0 & 0 & -1000
\end{array}\right] \\
B=\left[\begin{array}{c}
0 \\
0 \\
32770
\end{array}\right] \\
C=\left[\begin{array}{ccc}
28710 & 0 & 0
\end{array}\right] \\
D=[0]
\end{gathered}
$$

(Ecuación 6)

El procedimiento para designar la ubicación de los polos es el siguiente.

1) Se determina el polinomio característico de la matriz A para determinar los coeficientes:

$$
\begin{gathered}
\Delta(s)=(s I-A) \\
\Delta(s)=s^{3}+2 * 10^{3} s^{2}+1 * 10^{6} s \\
a_{0}=1 ; a_{1}=2 * 10^{3} ; \\
a_{2}=1 * 10^{6} ; a_{3}=0 \\
\text { (Ecuación } 7)
\end{gathered}
$$

2) Se determina la ubicación de los polos deseados, y su polinomio característico con sus coeficientes.
Para la ubicación de los polos se tienen en cuenta los siguientes aspectos:

- El tiempo de establecimiento (ts), que corresponde al tiempo que toma la señal en entrar en una oscilación igual al $1 \%$ del valor estacionario (Rairán, 2007).

- El sobrepico (Mp), que expresa la diferencia entre el punto más alto de la señal de salida y el valor de estado estacionario (Rairán, 2007).

- El tiempo de elevación (tr), que equivale al tiempo que la señal tarda en pasar del $10 \%$ al $90 \%$ del valor de estado estacionario (Rairán, 2007).

Se usa la función rlocus para observar en el plano $\mathrm{S}$, en donde se pueden ubicar los polos de tal manera que se cumplan los requerimientos de (ts, Mp y tr), usando los criterios de la ecuación 8.

Se eligen como criterios de diseño:

$$
\begin{aligned}
& t s=1[s] ; \quad \operatorname{tr}=0.3[s] \\
& M p=0.0001=0.01 \%
\end{aligned}
$$

\section{(Ecuación 8)}

En la figura 12 se observa el rlocus (plano s) aplicado al sistema (P2I), sobre el que se decide la ubicación de los polos. Allí, la línea vertical da la restricción por tiempo de estabilización (ts), las líneas diagonales dan la restricción por sobrepico (Mp), y el semicírculo da la restricción por tiempo de elevación. 


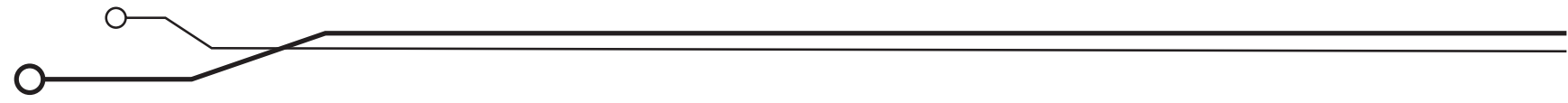

Figura 12. Rlocus para el modelo de avance

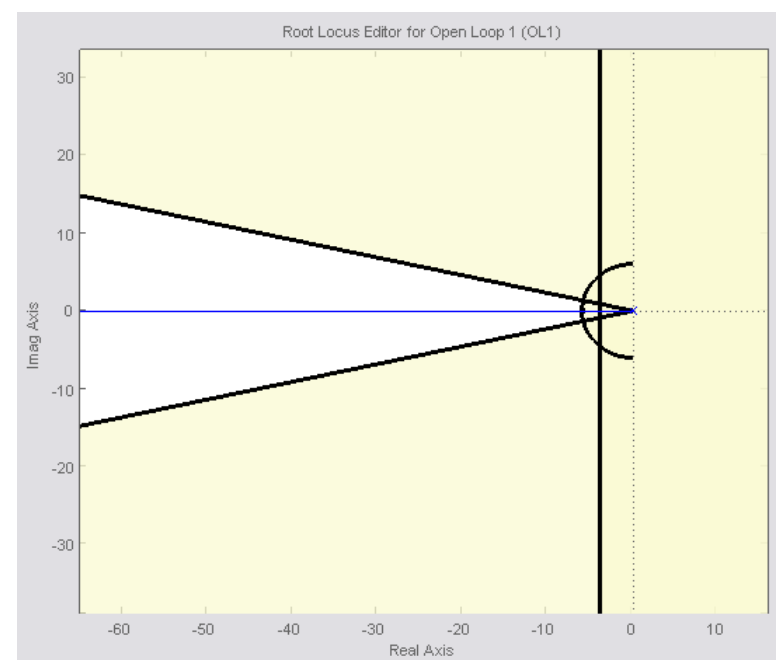

Fuente: elaboración propia

Es importante cumplir con los tres criterios de respuesta, por tanto, debe garantizarse la ubicación de los polos en la zona de color blanco.

Como se requiere que el máximo sobreimpulso sea cero, se escogen los siguientes polos reales:

$$
\begin{gathered}
s_{1}=-100 ; s_{2}=-200 \\
s_{3}=-300 \\
s^{3}+600 s^{2}+110 * 10^{3} s+6 * 10^{6} \\
\alpha_{0}=1 ; \alpha_{1}=600 ; \\
\alpha_{2}=110 * 10^{3} \alpha_{3}=6 * 10^{6}
\end{gathered}
$$

(Ecuación 9)

3) Se determinan los valores de las matrices $W$ y M para hallar la matriz de transformación T:

$$
W=\left[\begin{array}{ccc}
1 * 10^{6} & 2 * 10^{3} & 1 \\
2 * 10^{3} & 1 & 0 \\
1 & 0 & 0
\end{array}\right]
$$

$$
M=\left[\begin{array}{ccc}
0 & 0 & 32770 \\
0 & 32770 & -6.554 e+007 \\
32770 & -3.277 * 10^{7} & 3.277 * 10^{10}
\end{array}\right]
$$

(Ecuación 11)

$$
T=\left[\begin{array}{ccc}
32770 & 0 & 0 \\
0 & 32770 & 0 \\
0 & 32770000 & 32770
\end{array}\right]
$$

\section{(Ecuación 12)}

4) Se determina la ganancia de realimentación:

$$
\begin{gathered}
k=\left[\alpha_{3}-a_{3} \alpha_{2}-a_{2} \alpha_{1}-a_{1}\right] * T^{-1} \\
k=\left[\begin{array}{lll}
183.09 & 15.563 & -0.042722
\end{array}\right]
\end{gathered}
$$

\section{(Ecuación 13)}

Al aplicar $k$ al sistema, se obtiene la respuesta detallada en la figura 13. Allí se ve que al realimentar el sistema, este se estabiliza en un valor alto. Es necesario agregar una ganancia de prealimentación, para que la respuesta de salida sea igual a la señal de control. 
Figura 13. Respuesta del modelo de avance con realimentación de estados

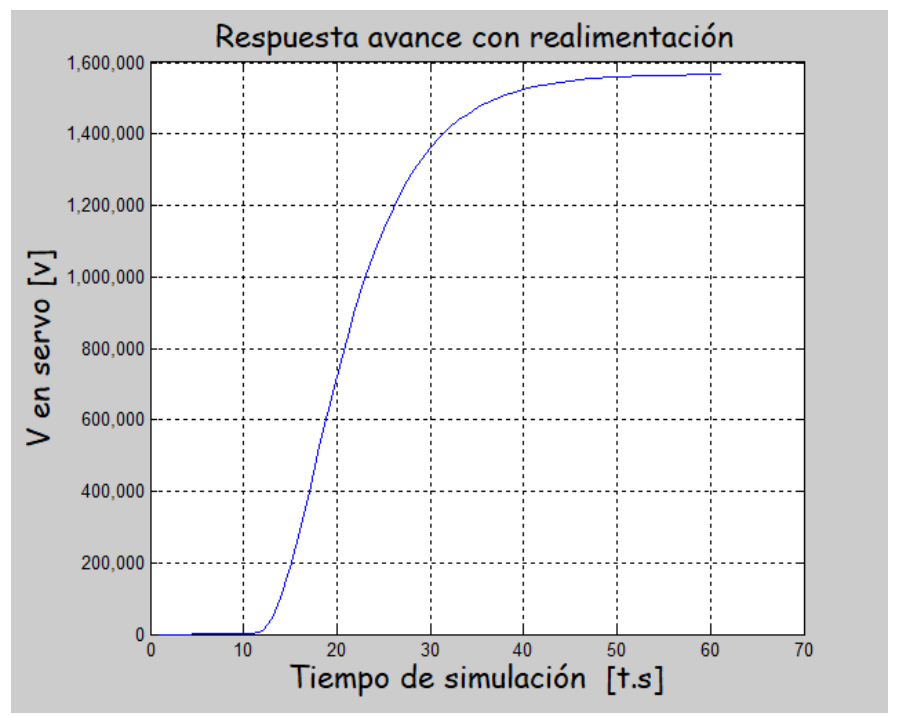

Fuente: elaboración propia

$$
\mathrm{Gp}=\frac{1}{1.57 * 10^{\wedge} 5}
$$

(Ecuación 14)

Figura 14. Respuesta del modelo de avance con realimentación de estados y prealimentación

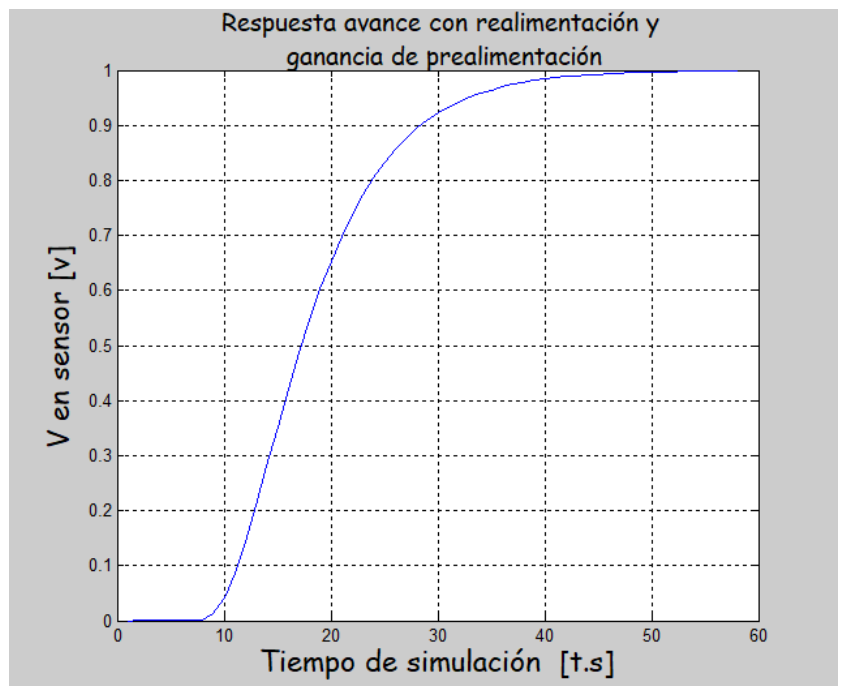

Fuente: elaboración propia

En la figura 14 se muestra la respuesta del sistema con prealimentación. El modelo con la matriz de realimentación $\mathrm{K}$ se muestra en la figura
15 , mientras que en la figura 16 se detalla el interior del modelo de avance. 


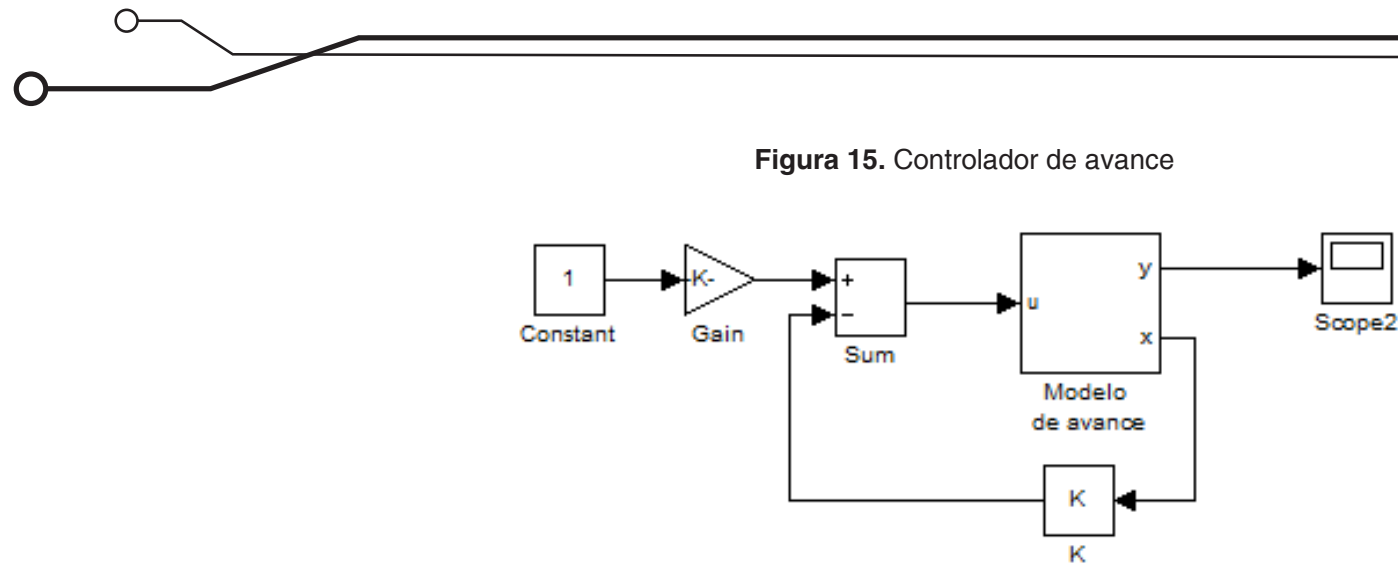

Fuente: elaboración propia

Figura 16. Modelo de avance

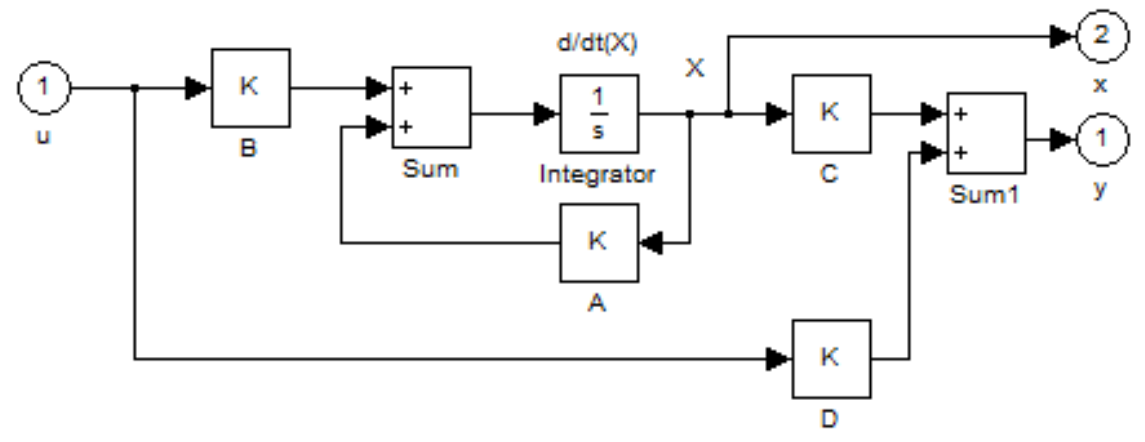

Fuente: elaboración propia

\section{Observador de estado (avance)}

En la práctica no se cuenta con los estados de realimentación $\mathrm{x}$, por lo que es indispensable diseñar un observador de estados (Katsuhiko, 1998), el cual toma la señal de salida (y), además de la señal de control $\mathrm{U}$, y proporciona los estados de realimentación, para multiplicarlos por la matriz de ganancias.

Como el observador es una copia de la planta, emplea la misma entrada y normalmente tiene la misma función de trasferencia, pero con un término extra compara la salida actual (y) con cierta salida estimada $(\hat{y})$. Así, los estados estimados se aproximan a los valores reales de los estados $\mathrm{x}$. Para diseñar el observador de estados de orden completo es necesario hallarle una ganancia de realimentación, así:
1) Se determina el polinomio característico de A, mediante la ecuación 7 .

2) Se determina el polinomio característico del observador.

$$
\begin{gathered}
s_{1 o}=-1000 ; s_{2 o}=-2000 \\
s_{3 o}=-3000 \\
\bar{a}_{0}=1 ; \bar{a}_{1}=6 * 10^{3} ; \bar{a}_{2}=11 * 10^{6} \\
\bar{a}_{3}=6 * 10^{9}
\end{gathered}
$$

(Ecuación 15) 
3) Se determina el $\mathrm{K}_{\mathrm{e}}$ ' del observador mediante la ecuación 16.

$$
\mathrm{K}_{\mathrm{e}}{ }^{\prime}=\left[\overline{\mathrm{a}_{1}}-\mathrm{a}_{1} \overline{\mathrm{a}_{2}}-\mathrm{a}_{2} \overline{\mathrm{a}_{3}}-\mathrm{a}_{3}\right]
$$

\section{(Ecuación 16)}

4) Se determina la matriz de transformación equivalente.

$$
T^{-1}=\left[\begin{array}{ccc}
1 & 0 & 0 \\
a_{1} & 1 & 0 \\
a_{2} & a_{1} & 1
\end{array}\right]\left[\begin{array}{c}
0 \\
C A \\
C A^{2}
\end{array}\right]
$$

(Ecuación 17)

5) Se calcula el $K_{e}^{\prime}$ aplicando las ecuaciones, desde la 18 hasta la 21.

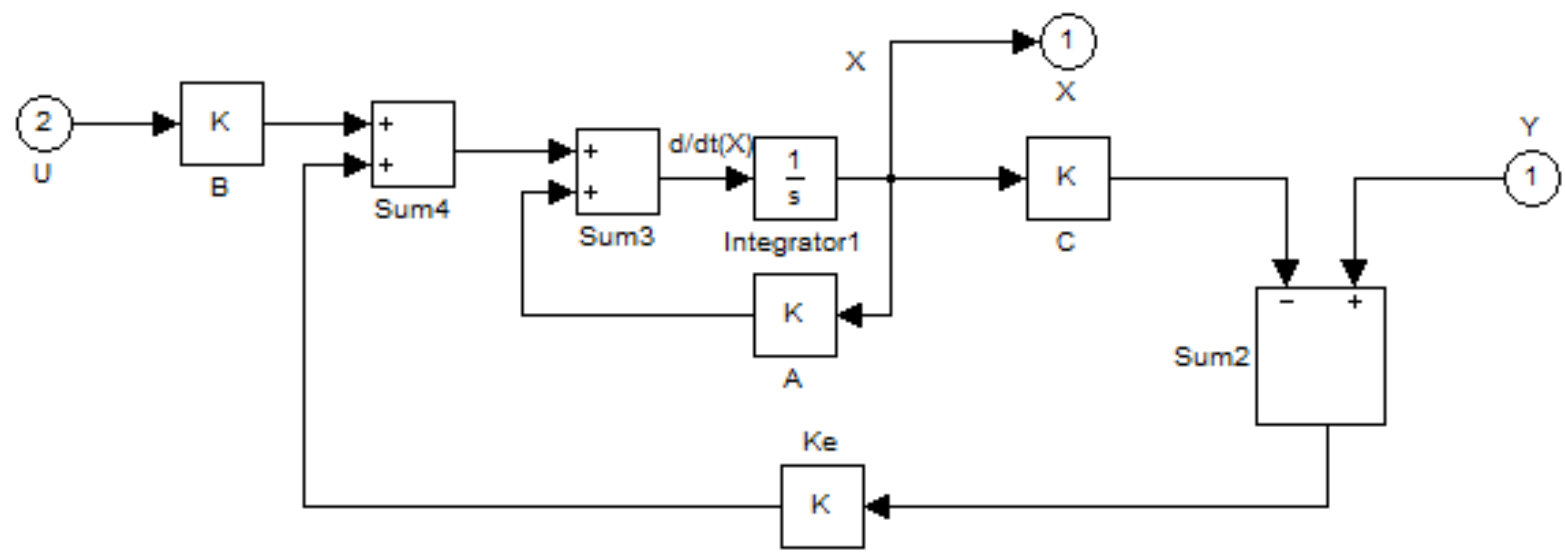

Fuente: elaboración propia

$$
\mathrm{K}_{\mathrm{e}}=\mathrm{K}_{\mathrm{e}}^{\prime} T^{-1}
$$

(Ecuación 18)

$$
N c=\left[\begin{array}{llll}
C & C A & C
\end{array}\right]
$$

(Ecuación 19)

$$
\begin{gathered}
T=N c * W \\
(\text { Ecuación 20) }
\end{gathered}
$$

$$
K e=\left[\begin{array}{c}
0.1393 \\
69.6621 \\
0
\end{array}\right]
$$

(Ecuación 21)

En la figura 17 se presenta un diagrama del interior del observador de estados:
Figura 17. Observador de estados 


\section{$26 \circlearrowright$ Revista de investigación \#ashtag}

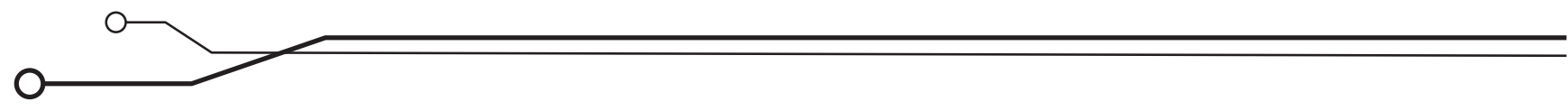

En la figura 18 se muestra el modelo con el observador adaptado.

Figura 18. Modelo de avance con el observador de estados

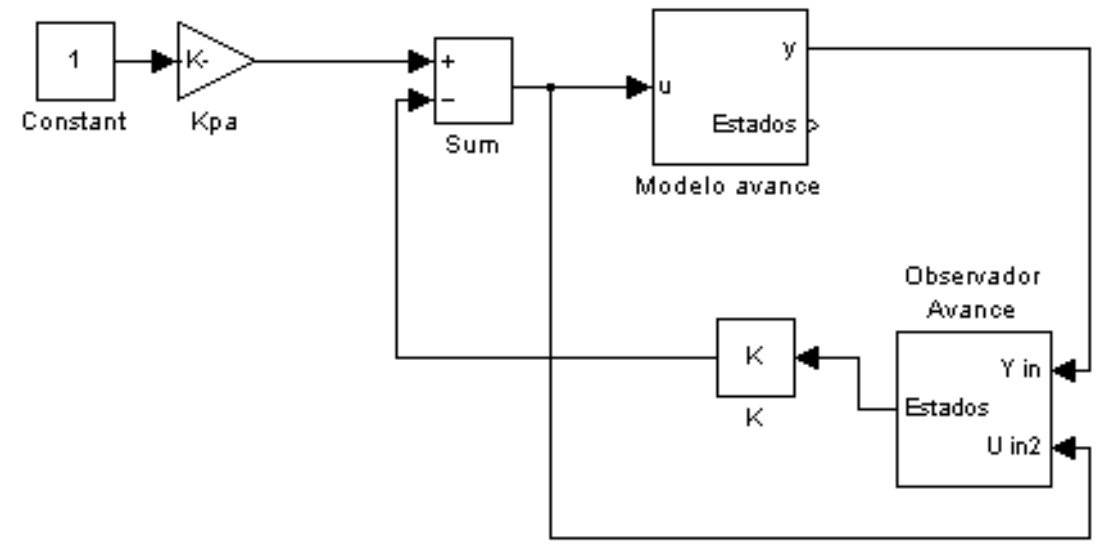

Fuente: elaboración propia

Para ver que el observador funciona correctamente, se realizan dos gráficas. La primera, en la figura 19, muestra un estado de la señal estados en el bloque observador, y el mismo estado de la señal estados del bloque modelo, que son iguales.

Figura 19. Comparación de un estado en el modelo y en el observador
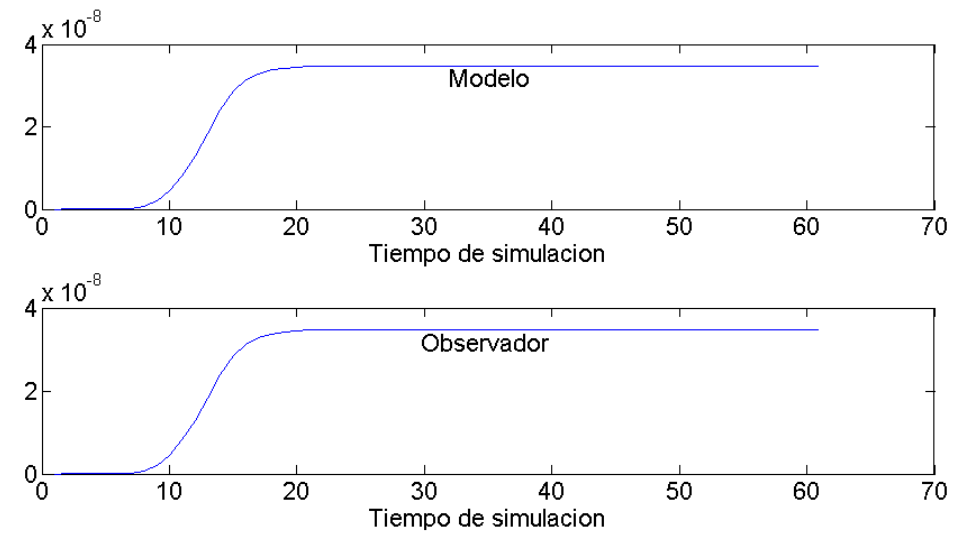

Fuente: elaboración propia

En la segunda de dichas gráficas (figura 20), se observa la señal de salida tanto del observador como del modelo. 
Figura 20. Comparación de la señal de salida del modelo y del observador

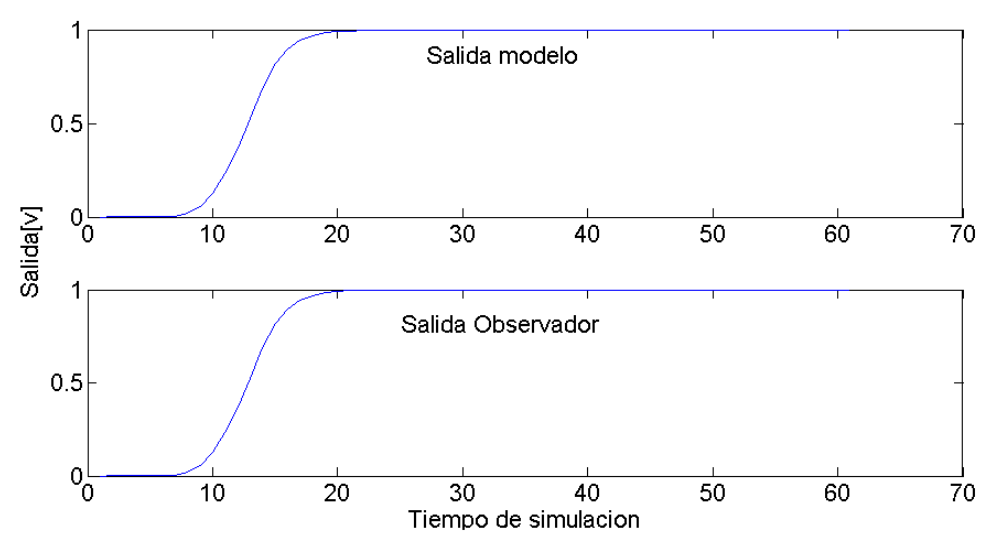

Fuente: elaboración propia

\section{Modelo de retroceso}

Para este otro modelo se determinan las matrices de igual manera como se hizo para el modelo de avance:

$$
\begin{gathered}
A=\left[\begin{array}{ccc}
0 & 1 & 0 \\
0 & -985.07 & 1 \\
0 & 0 & -999.87
\end{array}\right] \\
B=\left[\begin{array}{c}
0 \\
0 \\
16384
\end{array}\right] \\
C=\left[\begin{array}{lll}
12990 & 0 & 0
\end{array}\right] \\
D=[0]
\end{gathered}
$$

(Ecuación 22)

El procedimiento para designar la ubicación de los polos es el siguiente:

1) Se determina el polinomio característico de la matriz A para determinar los coeficientes:

$$
\begin{gathered}
\Delta(s)=(s I-A) \\
\Delta(s)=s^{3}+1985 s^{2}+9.849 * 10^{5} s \\
a_{0}=1 ; a_{1}=1985 ; \\
a_{2}=9.849 * 10^{5} ; a_{3}=0
\end{gathered}
$$

(Ecuación 23)

2) Se determina la ubicación de los polos deseados, y su polinomio característico con sus coeficientes:

Para la ubicación de los polos se tienen en cuenta los valores de la ecuación (8). Los polos seleccionados son los mismos que se indican en la ecuación (9).

3) Se determinan las matrices W, M y T:

$$
W=\left[\begin{array}{ccc}
9.8494 * 10^{5} & 1984.9 & 1 \\
1984.9 & 1 & 0 \\
1 & 0 & 0
\end{array}\right]
$$

(Ecuación 24) 


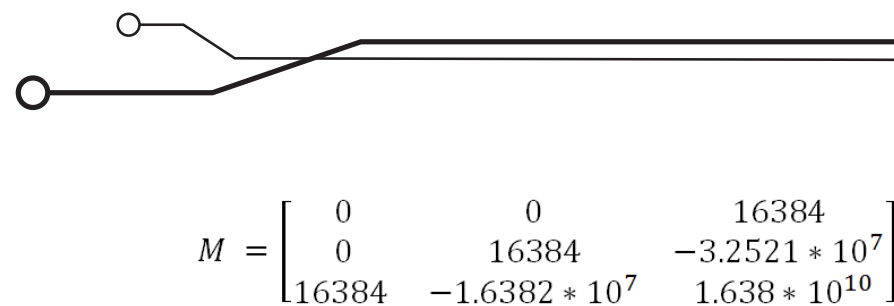

(Ecuación 25)

$$
T=\left[\begin{array}{ccc}
16384 & 0 & 0 \\
132.95 & 16384 & 0 \\
-941.45 & 1.6139 * 10^{7} & 16384
\end{array}\right]
$$

4) Se determina la ganancia de realimentación:

$$
\begin{aligned}
& k=\left[\begin{array}{lll}
\alpha_{3}-a_{3} \alpha_{2}-a_{2} \alpha_{1}-a_{1}
\end{array}\right] * T^{-1} \\
& k=\left[\begin{array}{lll}
365.96 & 29.872 & -0.084534
\end{array}\right]
\end{aligned}
$$

(Ecuación 27)

$\mathrm{Al}$ aplicar $\mathrm{k}$ al sistema se obtiene la siguiente respuesta:

Figura 21. Respuesta del modelo de retroceso con realimentación de estados

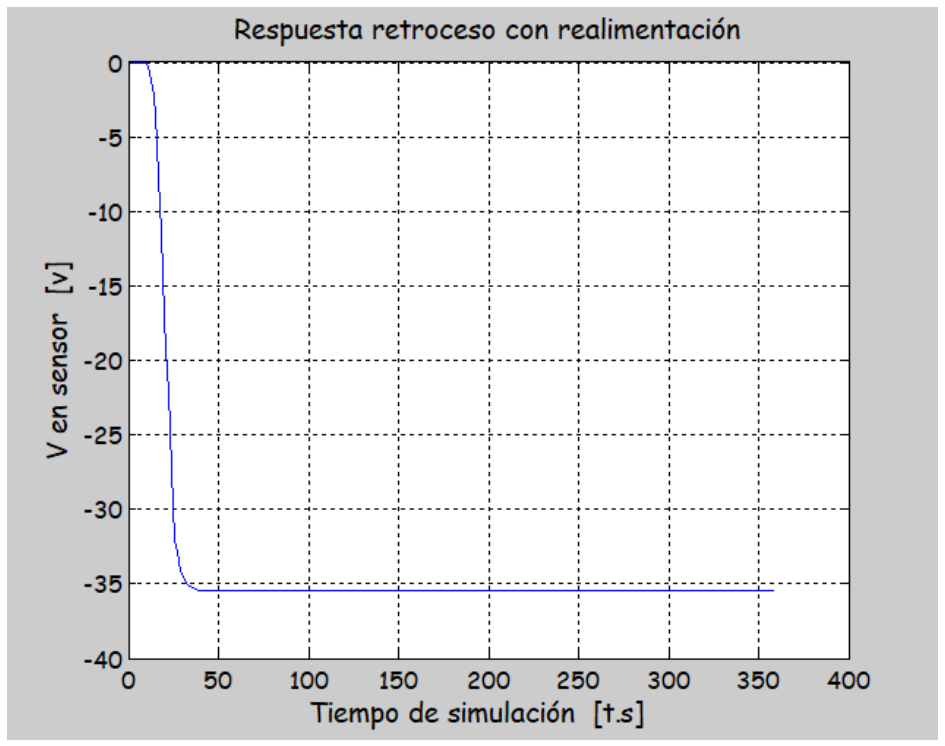

Fuente: elaboración propia

En la figura 21 se nota que, al realimentar el sistema, este se estabiliza en un valor alto. Por lo tanto es necesario agregar una ganancia de realimentación de tal manera que la respuesta de salida sea igual a la señal de control.

$$
G p=\frac{1}{35.5}
$$

(Ecuación 28)
En la figura 22 se nota que al aplicarle una ganancia de prealimentación al sistema se obtiene la respuesta deseada que sigue la señal de referencia de $-1 \mathrm{v}$.

El modelo con la matriz de realimentación $\mathrm{K}$ en Simulink es igual al de la figura 15, cambia es el valor de las matrices que caracterizan el comportamiento del sistema en retroceso. 
Figura 22. Respuesta del modelo de retroceso con realimentación de estados y prealimentación

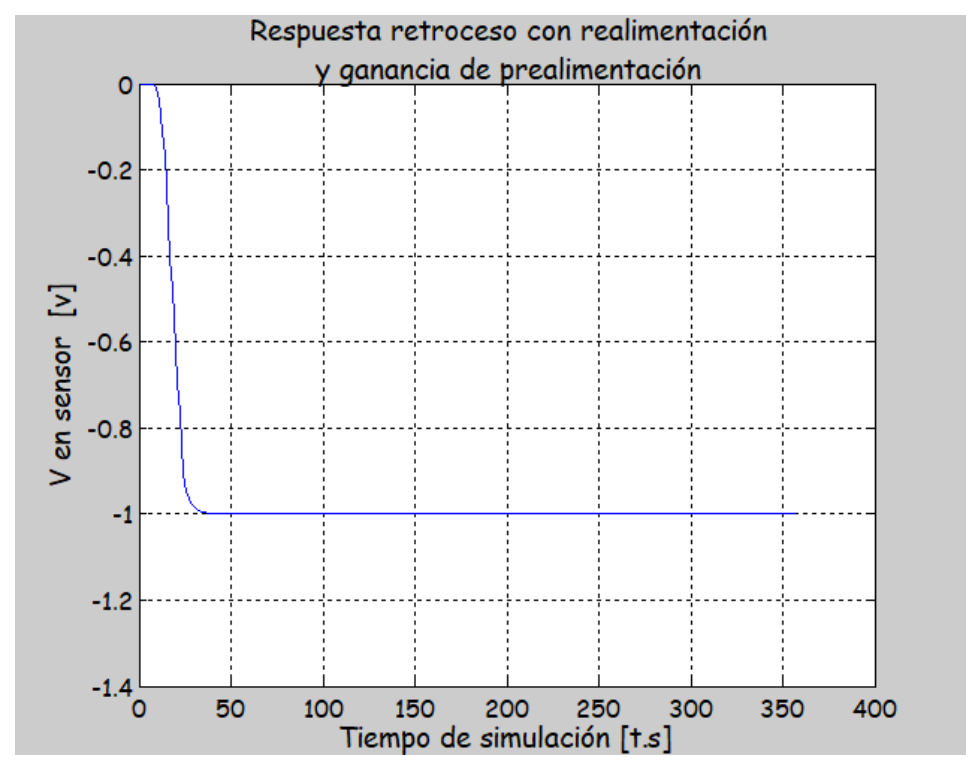

Fuente: elaboración propia

\section{Observador de estado (retroceso)}

Para diseñar el observador de estados para el modelo de retroceso, es necesario hallar una ganancia de realimentación para el observador de estados. Se calcula Nc y luego se calcula el valor de $\mathrm{T}$ para calcular el Ke, empleando el mismo procedimiento aplicado en el diseño del observador de estados para el modelo de avance.

$$
N C=\left[C C A C A^{2}\right]
$$

(Ecuación 29)

$$
\begin{gathered}
T=N c * W \\
(\text { Ecuación 30) } \\
K e=\left[\begin{array}{c}
-0.014242 \\
34.851 \\
-4653
\end{array}\right] \\
\text { (Ecuación 31) }
\end{gathered}
$$

Los esquemas para el modelo de retroceso son similares a las figuras $15,16,17$ y 18 , aunque las matrices del sistema son diferentes.

El modelo total del sistema, avance y retroceso combinados, presenta un error promedio de $9 \%$ con respecto a la respuesta real del sistema ante las señales cuadrada, diente de sierra y triangular. obtuvo un porcentaje de coincidencia de identificación aproximado de $93 \%$, usando como referencia una señal cuadrada. 


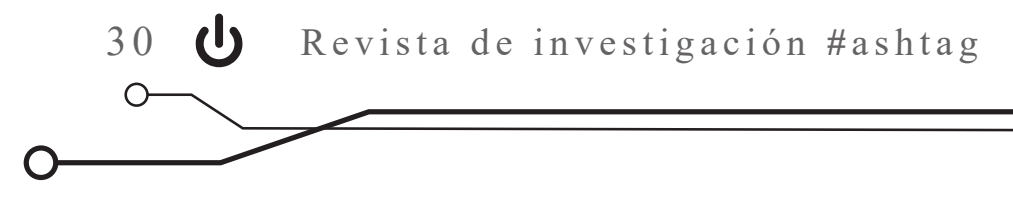

Se implementó un controlador por realimentación de estados para cada uno de los modelos estimados, cumpliendo siempre en la simulación con los parámetros de diseño establecidos. Sin embargo, una vez realizadas las pruebas sobre la

\section{Conclusiones}

Para seleccionar un modelo adecuado para iniciar la identificación del sistema, es necesario conocer muy bien el tipo de respuesta entregada por el sistema ante una entrada arbitraria. Sin embargo, es necesario además realizar fases de prueba y error para encontrar el modelo que más se ajuste al sistema real.

La identificación de sistemas se realiza generalmente con un único par de señales de entrada y salida, por lo que es posible que sistemas de ordenes diferentes entreguen respuestas similares ante una misma entrada, resultando así modelos con altos porcentajes de coincidencia. Pero, en ocasiones, estos modelos no describen correctamente el comportamiento real del sistema. Lo anterior implica que se deben realizar múltiples fases de identificación ante entradas diferentes para reducir el error producido.

La manera más adecuada de identificar un modelo es aplicar señales tipo escalón como lo son la señal cuadrada y la señal random, dado que tienen cambios buscos de nivel. La identificación realizada con la señal cuadrada caracteriza el sistema hidráulico, porque el modelo responde planta real y el sistema de adquisición de datos, se presentó un error del $20 \%$, debido principalmente a los tiempos de retardo de la comunicación USB.

de forma similar al comportamiento de la planta y los porcentajes de coincidencia en el Toolbox de identificación son altos.

La herramienta de identificación de sistemas utilizada permite identificar sistemas estrictamente lineales, por lo cual las no linealidades conocidas del sistema tuvieron que ser tenidas en cuenta después del proceso de identificación, lo cual aumentó el error en la estimación del modelo del sistema.

Al utilizar una tarjeta de adquisición de datos USB, se presenta un tiempo muerto en la comunicación, el cual produce un retardo de la señal de control hacia el sistema. Dependiendo del sistema que se esté controlando dicho retardo puede comprometer el funcionamiento en tiempo real del sistema de control.

El controlador calculado por medio de realimentación de estados y localización de polos presenta un error en la simulación de orden aceptable, por lo que cumple con los criterios de diseño tales como máximo sobre-impulso, tiempo de estabilización y levantamiento. 


\section{Recomendaciones}

Es posible utilizar otros métodos de identificación de sistemas, que tengan en cuenta algunas de las no linealidades presentes en el sistema, tales como banda muerta y saturación. Una posibilidad es utilizar algoritmos genéticos como método de optimización, considerando que su función objetivo puede contener no linealidades.
Se pueden utilizar control óptimo para el cálculo de las constantes de realimentación, o también técnicas de inteligencia artificial como redes neuronales, lógica difusa y algoritmos genéticos.

Es necesario utilizar un medio diferente de adquisición de datos para lograr control en tiempo real propiamente dicho, por ejemplo, una tarjeta de adquisición de datos por puerto PCI.

\section{Referencias}

Katsuhiko, O. (1998). Ingeniería de control moderna. Minessota: Universidad de Minnesota, Prentice Hall.

Ljung, L. (2008). System Identification Toolbox 7: Getting Started Guide. Nueva York: The MathWorks.

Martínez, M. y Rairán, D. (2001). Control de posición de un sistema hidráulico mediante un controlador PID digital. Tecnura, 5(9), 28-36.

MathWorks. Accelerating the Pace of Engineering and Science [recurso en línea]. Recuperado de https:// bit.ly/2QsPxxB

National Instruments. (2009). NI USB-621x User Manual. 2009. Austin, Texas: National Instruments Corporation. Recuperado de https:/ / bit.ly/3jncSNY

Rairán, J. (2007). Análisis de sistemas dinámicos y control PID. Bogotá: Universidad Distrital Francisco José de Caldas.

Rairán, J., Guerrero, C. y Mateus, J. (2010). Diseño de controladores de tipo proporcional integral derivativo (PID) y difuso para la posición de un motor de corriente continua (DC). Ingeniería y Universidad, 14(1), 137-160. Recuperado de https:/ / dialnet.unirioja.es/servlet/ articulo? codigo $=3243802$

Rairán, J. y Urrego, L. (2006). Implementación de un controlador difuso para la regulación de posición de un cilindro hidráulico lineal. Tecnura, 10(19), 18-28. Recuperado de https:// revistas.udistrital.edu.co/index.php/Tecnura/article/view/6212/7734 